\title{
Some properties of relative efficiency of estimators in a two linear regression equations system with identical parameter vectors
}

\section{Jibo $\mathrm{Wu}^{*}$}

"Correspondence:

linfen52@126.com

Department of Mathematics and

KLDAIP, Chongqing University of

Arts and Sciences, Chongqing,

402160, China

School of Mathematics and

Finances, Chongqing University of

Arts and Sciences, Chongqing,

402160, China

\begin{abstract}
Two normal linear models with some of the parameters identical are discussed in this article. We introduce four relative efficiencies to define the efficiency of estimator in two linear regression equations system with identical parameter vectors, also we give the lower and upper bounds of the four relative efficiencies.
\end{abstract}

Keywords: best linear unbiased estimator; common parameter; relative efficiency

\section{Introduction}

Consider a system $(\mathrm{H})$ formed by two linear models:

$$
\begin{aligned}
& y_{1}=X_{1} \beta+Z_{1} \beta_{1}+\varepsilon_{1}, \\
& y_{2}=X_{2} \beta+Z_{2} \beta_{2}+\varepsilon_{2},
\end{aligned}
$$

where for $i=1,2, y_{i}$ is $n_{i} \times 1$ vector of observations, $X_{i}$ and $Z_{i}$ are $n_{i} \times p$ and $n_{i} \times t_{i}$ full rank matrices satisfying $\operatorname{rank}\left(X_{i}, Z_{i}\right)=\operatorname{rank}\left(X_{i}\right)+\operatorname{rank}\left(Z_{i}\right)$ with $\operatorname{rank}(\cdot)$ denoting the rank of a matrix, $\beta$ and $\beta_{i}$ are $p \times 1$ and $t_{i} \times 1$ unknown parameters, $\varepsilon_{i}$ is $n_{i} \times 1$ random vector supposed to follow a multivariate normal distribution mean 0 and variance covariance matrix $\sigma_{i} I, \sigma_{i}$ being a known parameter, $\varepsilon_{1}$ and $\varepsilon_{2}$ are independent.

Define $Q_{i}=I-Z_{i}\left(Z_{i}^{\prime} Z_{i}\right)^{-1} Z_{i}^{\prime}, T_{i}=\left(Z_{i}^{\prime} Z_{i}\right)^{-1} Z_{i}^{\prime} X_{i}$ and $r=\frac{\sigma_{1}}{\sigma_{2}}$. Then by Liu [1] we have the following:

(1) In the single equation (1), the best linear unbiased estimators (BLUE) of $\beta$ and $\beta_{1}$ are given respectively by

$$
\begin{aligned}
& \hat{\beta}=\left(X_{1}^{\prime} Q_{1} X_{1}\right)^{-1} X_{1}^{\prime} Q_{1} y_{1}, \\
& \hat{\beta}_{1}=\left(Z_{1}^{\prime} Z_{1}\right)^{-1} Z_{1}^{\prime} y_{1}-T_{1} \hat{\beta} .
\end{aligned}
$$

(2) In the single equation (2), the best linear unbiased estimators (BLUE) of $\beta$ and $\beta_{2}$ are given respectively by

$$
\begin{aligned}
& \tilde{\beta}=\left(X_{2}^{\prime} Q_{2} X_{2}\right)^{-1} X_{2}^{\prime} Q_{2} y_{2}, \\
& \tilde{\beta}_{2}=\left(Z_{2}^{\prime} Z_{2}\right)^{-1} Z_{2}^{\prime} y_{2}-T_{2} \tilde{\beta} .
\end{aligned}
$$


(3) For the system (H), the BLUE of $\beta, \beta_{1}$ and $\beta_{2}$ are given respectively by

$$
\begin{aligned}
& \beta^{*}(r)=\left(X_{1}^{\prime} Q_{1} X_{1}+r X_{2}^{\prime} Q_{2} X_{2}\right)^{-1}\left(X_{1}^{\prime} Q_{1} y_{1}+r X_{2}^{\prime} Q_{2} y_{2}\right), \\
& \beta_{1}^{*}=\left(Z_{1}^{\prime} Z_{1}\right)^{-1} Z_{1}^{\prime} y_{1}-T_{1} \beta^{*}(r), \\
& \beta_{2}^{*}=\left(Z_{2}^{\prime} Z_{2}\right)^{-1} Z_{2}^{\prime} y_{2}-T_{2} \beta^{*}(r) .
\end{aligned}
$$

In this article, we only discuss the estimation of the parameter $\beta$. Liu [1] gave the comparison between the estimators $\hat{\beta}, \tilde{\beta}$ and $\beta^{*}(r)$ in the mean squared error criterion when $\sigma_{i}$ are known. He also gave an estimator when $\sigma_{i}$ are unknown and discussed the statistical properties of the estimators $\hat{\beta}, \tilde{\beta}$ and $\beta^{*}(r)$. Ma and Wang [2] also studied the estimators $\hat{\beta}, \tilde{\beta}$ and $\beta^{*}(r)$ in the mean squared error criterion.

It is easy to compute that

$$
\begin{aligned}
& \operatorname{Cov}(\hat{\beta})=\sigma_{1}\left(X_{1}^{\prime} Q_{1} X_{1}\right)^{-1}, \\
& \operatorname{Cov}(\tilde{\beta})=\sigma_{2}\left(X_{2}^{\prime} Q_{2} X_{2}\right)^{-1}, \\
& \operatorname{Cov}\left(\beta^{*}(r)\right)=\sigma_{1}\left(X_{1}^{\prime} Q_{1} X_{1}+r X_{2}^{\prime} Q_{2} X_{2}\right)^{-1} .
\end{aligned}
$$

From Equations (10)-(12), we can see that

$$
\operatorname{Cov}\left(\beta^{*}(r)\right) \leq \operatorname{Cov}(\hat{\beta}), \quad \operatorname{Cov}\left(\beta^{*}(r)\right) \leq \operatorname{Cov}(\tilde{\beta})
$$

In practice, $\sigma_{i}$ may be unknown, in this case we can use $\hat{\beta}$ or $\tilde{\beta}$ to replace $\beta^{*}(r)$. However, this will lead to loss, we introduce the relative efficiency to define the loss. Relative efficiency has been studied by many researchers such as Yang [3], Wang and Ip [4], Liu et al. [5, 6], Yang and Wang [7], Wang and Yang [8, 9] and Yang and Wu [10].

In this article, we introduce four relative efficiencies in system $(\mathrm{H})$, and we also give the lower and upper bounds of the four relative efficiencies.

The rest of the article is organized as follows. In Section 2, we propose the new relative efficiency. Sections 3 and 4 give the lower and upper bounds of the relative efficiencies proposed in Section 2. Some concluding remarks are given in Section 5.

\section{New relative efficiency}

In order to define the loss when we use $\hat{\beta}$ or $\tilde{\beta}$ to replace $\beta^{*}(r)$, we introduce four relative efficiencies as follows:

$$
\begin{aligned}
& e_{1}\left(\beta^{*}(r) \mid \hat{\beta}\right)=\frac{\left|\operatorname{Cov}\left(\beta^{*}(r)\right)\right|}{|\operatorname{Cov}(\hat{\beta})|}, \\
& e_{2}\left(\beta^{*}(r) \mid \tilde{\beta}\right)=\frac{\left|\operatorname{Cov}\left(\beta^{*}(r)\right)\right|}{|\operatorname{Cov}(\tilde{\beta})|}, \\
& e_{3}\left(\beta^{*}(r) \mid \hat{\beta}\right)=\frac{\operatorname{tr}\left(\operatorname{Cov}\left(\beta^{*}(r)\right)\right)}{\operatorname{tr}(\operatorname{Cov}(\hat{\beta}))}, \\
& e_{4}\left(\beta^{*}(r) \mid \tilde{\beta}\right)=\frac{\operatorname{tr}\left(\operatorname{Cov}\left(\beta^{*}(r)\right)\right)}{\operatorname{tr}(\operatorname{Cov}(\tilde{\beta}))},
\end{aligned}
$$


where $|A|$ and $\operatorname{tr}(A)$ denote the determinant and trace of matrix $A$, respectively. By Equation (13), we have $0<e_{i}(\cdot \cdot \cdot) \leq 1, i=1,2,3,4$. In the next section we will give the lower and upper bounds of $e_{1}\left(\beta^{*}(r) \mid \hat{\beta}\right)$ and $e_{2}\left(\beta^{*}(r) \mid \tilde{\beta}\right)$.

\section{The lower and upper bounds of $e_{1}\left(\beta^{*}(r) \mid \hat{\beta}\right)$ and $e_{2}\left(\beta^{*}(r) \mid \tilde{\beta}\right)$}

In this section we give the lower and upper bounds of $e_{1}\left(\beta^{*}(r) \mid \hat{\beta}\right)$ and $e_{2}\left(\beta^{*}(r) \mid \tilde{\beta}\right)$. Firstly, we give some lemmas and notations which are needed in the following discussion. Let $A$ be an $n \times n$ nonnegative definite matrix, $\lambda_{1}(A) \geq \lambda_{2}(A) \geq \cdots \geq \lambda_{n}(A)$ stands for the ordered eigenvalues of matrix $A$.

Lemma 3.1 [11] Let $A$ be an $n \times n$ nonnegative definite matrix, and let $B$ be an $n \times n$ nonnegative definite matrix, then we have

$$
\lambda_{n}(A) \lambda_{i}(B) \leq \lambda_{i}(A B) \leq \lambda_{1}(A) \lambda_{i}(B), \quad i=1,2, \ldots, n .
$$

Lemma 3.2 [12] Let $\Delta_{1}=\operatorname{diag}\left(\tau_{1}, \tau_{2}, \ldots, \tau_{p}\right), \tau_{1} \geq \tau_{2} \geq \cdots \geq \tau_{p}>0, \Delta_{2}=\operatorname{diag}\left(\mu_{1}, \mu_{2}\right.$, $\left.\ldots, \mu_{p}\right), \mu_{1} \geq \mu_{2} \geq \cdots \geq \mu_{p} \geq 0$ and $A$ be an $p \times p$ orthogonal matrix, then we have

$$
\sum_{i=1}^{p} \tau_{i} \mu_{p+1-i} \leq \operatorname{tr}\left(\Delta_{1} A^{\prime} \Delta_{2} A\right) \leq \sum_{i=1}^{p} \tau_{i} \mu_{i}
$$

Now we will give the lower and upper bounds of $e_{1}\left(\beta^{*}(r) \mid \hat{\beta}\right)$.

Theorem 3.1 Let $\beta^{*}(r)$ and $\hat{\beta}$ be given in Equations (7) and (3), let $e_{1}\left(\beta^{*}(r) \mid \hat{\beta}\right)$ be defined in Equation (14), then we have

$$
\frac{1}{\prod_{i=1}^{p}\left(1+r \theta_{p}^{-1} \eta_{i}\right)} \leq e_{1}\left(\beta^{*}(r) \mid \hat{\beta}\right) \leq \frac{1}{\prod_{i=1}^{p}\left(1+r \theta_{1}^{-1} \eta_{i}\right)}
$$

where $\theta_{1} \geq \cdots \geq \theta_{p}$ is the ordered eigenvalues of $X_{1}^{\prime} Q_{1} X_{1}, \eta_{1} \geq \cdots \geq \eta_{p}$ is the ordered eigenvalues of $X_{2}^{\prime} Q_{2} X_{2}$.

Proof By the definition of $e_{1}\left(\beta^{*}(r) \mid \hat{\beta}\right)$, we have

$$
\begin{aligned}
e_{1}\left(\beta^{*}(r) \mid \hat{\beta}\right) & =\frac{\left|\operatorname{Cov}\left(\beta^{*}(r)\right)\right|}{|\operatorname{Cov}(\hat{\beta})|} \\
& =\frac{\left|\sigma_{1}\left(X_{1}^{\prime} Q_{1} X_{1}+r X_{2}^{\prime} Q_{2} X_{2}\right)^{-1}\right|}{\left|\sigma_{1}\left(X_{1}^{\prime} Q_{1} X_{1}\right)^{-1}\right|} \\
& =\frac{\left|X_{1}^{\prime} Q_{1} X_{1}\right|}{\left|X_{1}^{\prime} Q_{1} X_{1}+r X_{2}^{\prime} Q_{2} X_{2}\right|} .
\end{aligned}
$$

It is easy to see that $X_{1}^{\prime} Q_{1} X_{1}>0$ and $X_{2}^{\prime} Q_{2} X_{2}>0$. Define

$$
A=\left(X_{1}^{\prime} Q_{1} X_{1}\right)^{-1 / 2}\left(X_{2}^{\prime} Q_{2} X_{2}\right)\left(X_{1}^{\prime} Q_{1} X_{1}\right)^{-1 / 2},
$$

then $A>0$, there exists an orthogonal matrix $N$ such that

$$
N A N^{\prime}=\operatorname{diag}\left(\zeta_{1}, \ldots, \zeta_{p}\right) \triangleq \Delta,
$$


where $\zeta_{1} \geq \cdots \geq \zeta_{p}$ is the eigenvalues of $A$. Now we define $M=N\left(X_{1}^{\prime} Q_{1} X_{1}\right)^{-1 / 2}$, then we have

$$
\begin{aligned}
M\left(X_{1}^{\prime} Q_{1} X_{1}\right) M^{\prime} & =N N^{\prime}=I_{p}, \\
M\left(X_{2}^{\prime} Q_{2} X_{2}\right) M^{\prime} & =N\left(X_{1}^{\prime} Q_{1} X_{1}\right)^{-1 / 2}\left(X_{2}^{\prime} Q_{2} X_{2}\right)\left(X_{1}^{\prime} Q_{1} X_{1}\right)^{-1 / 2} N^{\prime} \\
& =N A N^{\prime}=\Delta .
\end{aligned}
$$

Thus

$$
\begin{aligned}
& X_{1}^{\prime} Q_{1} X_{1}=M^{-1} M^{\prime-1}, \\
& X_{2}^{\prime} Q_{2} X_{2}=M^{-1} \Delta M^{\prime-1} .
\end{aligned}
$$

Then we put Equations (25) and (26) into Equation (21), and we have

$$
\begin{aligned}
e_{1}\left(\beta^{*}(r) \mid \hat{\beta}\right) & =\frac{\left|X_{1}^{\prime} Q_{1} X_{1}\right|}{\left|X_{1}^{\prime} Q_{1} X_{1}+r X_{2}^{\prime} Q_{2} X_{2}\right|} \\
& =\frac{\left|M^{-1} M^{\prime-1}\right|}{\left|M^{-1} M^{\prime-1}+r M^{-1} \Delta M^{\prime-1}\right|} \\
& =\frac{\left|M^{-1}\right|\left|M^{\prime-1}\right|}{\left|M^{-1}\right|\left|I_{p}+r \Delta\right|\left|M^{\prime-1}\right|}=\frac{1}{\left|I_{p}+r \Delta\right|} .
\end{aligned}
$$

Since $A=\left(X_{1}^{\prime} Q_{1} X_{1}\right)^{-1 / 2}\left(X_{2}^{\prime} Q_{2} X_{2}\right)\left(X_{1}^{\prime} Q_{1} X_{1}\right)^{-1 / 2}$ has the same eigenvalues of $\left(X_{2}^{\prime} Q_{2} X_{2}\right) \times$ $\left(X_{1}^{\prime} Q_{1} X_{1}\right)^{-1}$, we have $\lambda_{i}(A)=\lambda_{i}\left(\left(X_{2}^{\prime} Q_{2} X_{2}\right)\left(X_{1}^{\prime} Q_{1} X_{1}\right)^{-1}\right), i=1,2, \ldots, p$. Then by Lemma 3.1 we have

$$
\begin{aligned}
\lambda_{p}\left(\left(X_{1}^{\prime} Q_{1} X_{1}\right)^{-1}\right) \lambda_{i}\left(X_{2}^{\prime} Q_{2} X_{2}\right) & \leq \lambda_{i}\left(\left(X_{2}^{\prime} Q_{2} X_{2}\right)\left(X_{1}^{\prime} Q_{1} X_{1}\right)^{-1}\right) \\
& \leq \lambda_{1}\left(\left(X_{1}^{\prime} Q_{1} X_{1}\right)^{-1}\right) \lambda_{i}\left(X_{2}^{\prime} Q_{2} X_{2}\right) .
\end{aligned}
$$

On the other hand,

$$
\begin{aligned}
& \lambda_{p}\left(\left(X_{1}^{\prime} Q_{1} X_{1}\right)^{-1}\right)=\lambda_{1}^{-1}\left(X_{1}^{\prime} Q_{1} X_{1}\right)=\theta_{1}^{-1}, \\
& \lambda_{1}\left(\left(X_{1}^{\prime} Q_{1} X_{1}\right)^{-1}\right)=\lambda_{p}^{-1}\left(X_{1}^{\prime} Q_{1} X_{1}\right)=\theta_{p}^{-1},
\end{aligned}
$$

where $\theta_{1} \geq \cdots \geq \theta_{p}$ is the ordered eigenvalues of $X_{1}^{\prime} Q_{1} X_{1}$. By Equations (28)-(30), we obtain

$$
\theta_{1}^{-1} \eta_{i} \leq \lambda_{i}\left(\left(X_{2}^{\prime} Q_{2} X_{2}\right)\left(X_{1}^{\prime} Q_{1} X_{1}\right)^{-1}\right) \leq \theta_{p}^{-1} \eta_{i}, \quad i=1, \ldots, p,
$$

where $\eta_{1} \geq \cdots \geq \eta_{p}$ is the ordered eigenvalues of $X_{2}^{\prime} Q_{2} X_{2}$. Thus by Equations (27) and (31), we have

$$
\frac{1}{\prod_{i=1}^{p}\left(1+r \theta_{p}^{-1} \eta_{i}\right)} \leq e_{1}\left(\beta^{*}(r) \mid \hat{\beta}\right) \leq \frac{1}{\prod_{i=1}^{p}\left(1+r \theta_{1}^{-1} \eta_{i}\right)} .
$$


Corollary 3.1 Let $\beta^{*}(r)$ and $\hat{\beta}$ be given in Equations (7) and (3), let $e_{1}\left(\beta^{*}(r) \mid \hat{\beta}\right)$ be defined in Equation (14), $X_{1}^{\prime} Q_{1} X_{1}$ and $X_{2}^{\prime} Q_{2} X_{2}$ communicate, then we have

$$
\frac{\theta_{p}^{p}}{\left(\theta_{1}+r \eta_{1}\right)^{p}} \leq e_{1}\left(\beta^{*}(r) \mid \hat{\beta}\right) \leq \frac{\theta_{1}^{p}}{\left(\theta_{p}+r \eta_{p}\right)^{p}}
$$

where $\theta_{1} \geq \cdots \geq \theta_{p}$ is the ordered eigenvalues of $X_{1}^{\prime} Q_{1} X_{1}, \eta_{1} \geq \cdots \geq \eta_{p}$ is the ordered eigenvalues of $X_{2}^{\prime} Q_{2} X_{2}$.

Proof Since $X_{1}^{\prime} Q_{1} X_{1}$ and $X_{2}^{\prime} Q_{2} X_{2}$ communicate, there exists an orthogonal matrix $G$ such that

$$
\begin{aligned}
& G^{\prime} X_{1}^{\prime} Q_{1} X_{1} G=\operatorname{diag}\left(\theta_{1}, \ldots, \theta_{p}\right) \triangleq \Sigma, \\
& G^{\prime} X_{2}^{\prime} Q_{2} X_{2} G=\operatorname{diag}\left(\eta_{1}, \ldots, \eta_{p}\right) \triangleq \Omega,
\end{aligned}
$$

where $\theta_{1} \geq \cdots \geq \theta_{p}$ is the ordered eigenvalues of $X_{1}^{\prime} Q_{1} X_{1}, \eta_{1} \geq \cdots \geq \eta_{p}$ is the ordered eigenvalues of $X_{2}^{\prime} Q_{2} X_{2}$.

By the definition of $e_{1}\left(\beta^{*}(r) \mid \hat{\beta}\right)$, we have

$$
\begin{aligned}
e_{1}\left(\beta^{*}(r) \mid \hat{\beta}\right) & =\frac{\left|\operatorname{Cov}\left(\beta^{*}(r)\right)\right|}{|\operatorname{Cov}(\hat{\beta})|} \\
& =\frac{\left|X_{1}^{\prime} Q_{1} X_{1}\right|}{\left|X_{1}^{\prime} Q_{1} X_{1}+r X_{2}^{\prime} Q_{2} X_{2}\right|} \\
& =\frac{\left|G \Sigma G^{\prime}\right|}{\left|G \Sigma G^{\prime}+r G \Omega G^{\prime}\right|} \\
& =\frac{\prod_{i=1}^{p} \theta_{i}}{\prod_{i=1}^{p}\left(\theta_{i}+r \eta_{i}\right)} .
\end{aligned}
$$

Thus we have

$$
\frac{\theta_{p}^{p}}{\left(\theta_{1}+r \eta_{1}\right)^{p}} \leq e_{1}\left(\beta^{*}(r) \mid \hat{\beta}\right) \leq \frac{\theta_{1}^{p}}{\left(\theta_{p}+r \eta_{p}\right)^{p}} .
$$

Using the same way, we can give the lower and upper bounds of $e_{2}\left(\beta^{*}(r) \mid \tilde{\beta}\right)$.

Theorem 3.2 Let $\beta^{*}(r)$ and $\tilde{\beta}$ be given in Equations (7) and (5), let $e_{2}\left(\beta^{*}(r) \mid \tilde{\beta}\right)$ be defined in Equation (15), then we have

$$
\frac{1}{\prod_{i=1}^{p}\left(r+\eta_{p}^{-1} \theta_{i}\right)} \leq e_{2}\left(\beta^{*}(r) \mid \tilde{\beta}\right) \leq \frac{1}{\prod_{i=1}^{p}\left(r+\eta_{1}^{-1} \theta_{i}\right)},
$$

where $\theta_{1} \geq \cdots \geq \theta_{p}$ is the ordered eigenvalues of $X_{1}^{\prime} Q_{1} X_{1}, \eta_{1} \geq \cdots \geq \eta_{p}$ is the ordered eigenvalues of $X_{2}^{\prime} Q_{2} X_{2}$.

Corollary 3.2 Let $\beta^{*}(r)$ and $\tilde{\beta}$ be given in Equations (7) and (5), let $e_{2}\left(\beta^{*}(r) \mid \tilde{\beta}\right)$ be defined in Equation (15), $X_{1}^{\prime} Q_{1} X_{1}$ and $X_{2}^{\prime} Q_{2} X_{2}$ communicate, then we have

$$
\frac{\eta_{p}^{p}}{\left(\theta_{1}+r \eta_{1}\right)^{p}} \leq e_{2}\left(\beta^{*}(r) \mid \tilde{\beta}\right) \leq \frac{\eta_{1}^{p}}{\left(\theta_{p}+r \eta_{p}\right)^{p}} .
$$




\section{The lower and upper bounds of $e_{3}\left(\beta^{*}(r) \mid \hat{\beta}\right)$ and $e_{4}\left(\beta^{*}(r) \mid \tilde{\beta}\right)$}

In this section we give the lower and upper bounds of $e_{3}\left(\beta^{*}(r) \mid \hat{\beta}\right)$ and $e_{4}\left(\beta^{*}(r) \mid \tilde{\beta}\right)$. Firstly we give the lower and upper bounds of $e_{3}\left(\beta^{*}(r) \mid \hat{\beta}\right)$.

Theorem 4.1 Let $\beta^{*}(r)$ and $\hat{\beta}$ be given in Equations (7) and (3), let $e_{3}\left(\beta^{*}(r) \mid \hat{\beta}\right)$ be defined in Equation (16), then we have

$$
\frac{\sum_{i=1}^{p} \theta_{p+1-i}^{-1}\left(1+r \zeta_{i}\right)^{-1}}{\sum_{i=1}^{p} \theta_{i}^{-1}} \leq e_{3}\left(\beta^{*}(r) \mid \hat{\beta}\right) \leq \frac{\sum_{i=1}^{p} \theta_{p+1-i}^{-1}\left(1+r \zeta_{p+1-i}\right)^{-1}}{\sum_{i=1}^{p} \theta_{i}^{-1}},
$$

where $\theta_{1} \geq \cdots \geq \theta_{p}$ is the ordered eigenvalues of $X_{1}^{\prime} Q_{1} X_{1}, \zeta_{1} \geq \cdots \geq \zeta_{p}$ is the ordered eigenvalues of $\left(X_{1}^{\prime} Q_{1} X_{1}\right)^{-1 / 2}\left(X_{2}^{\prime} Q_{2} X_{2}\right)\left(X_{1}^{\prime} Q_{1} X_{1}\right)^{-1 / 2}$.

Proof Since $X_{1}^{\prime} Q_{1} X_{1}>0$, there exists an orthogonal matrix $K_{1}$ such that

$$
X_{1}^{\prime} Q_{1} X_{1}=K_{1}^{\prime} \Sigma K_{1}, \quad \Sigma=\operatorname{diag}\left(\theta_{1}, \ldots, \theta_{p}\right)
$$

where $\theta_{1} \geq \cdots \geq \theta_{p}$ is the ordered eigenvalues of $X_{1}^{\prime} Q_{1} X_{1}$. Similar to Theorem 3.1, we define

$$
A=\left(X_{1}^{\prime} Q_{1} X_{1}\right)^{-1 / 2}\left(X_{2}^{\prime} Q_{2} X_{2}\right)\left(X_{1}^{\prime} Q_{1} X_{1}\right)^{-1 / 2} \text {. }
$$

Since $A>0$, there exists an orthogonal matrix $K_{2}$ such that

$$
A=K_{2}^{\prime} \Delta K_{2}, \quad \Delta=\operatorname{diag}\left(\zeta_{1}, \ldots, \zeta_{p}\right),
$$

where $\zeta_{1} \geq \cdots \geq \zeta_{p}$ is the order eigenvalues of $A$.

We can easily compute that

$$
\operatorname{tr}(\operatorname{Cov}(\hat{\beta}))=\sigma_{1} \operatorname{tr}\left(\left(X_{1}^{\prime} Q_{1} X_{1}\right)^{-1}\right)=\sigma_{1} \sum_{i=1}^{p} \theta_{i}^{-1}
$$

and

$$
\begin{aligned}
\operatorname{tr}\left(\operatorname{Cov}\left(\beta^{*}(r)\right)\right) & =\sigma_{1} \operatorname{tr}\left(\left(X_{1}^{\prime} Q_{1} X_{1}+r X_{2}^{\prime} Q_{2} X_{2}\right)^{-1}\right) \\
& =\sigma_{1} \operatorname{tr}\left(\left(X_{1}^{\prime} Q_{1} X_{1}\right)^{-1 / 2}\left(I_{p}+r A\right)^{-1}\left(X_{1}^{\prime} Q_{1} X_{1}\right)^{-1 / 2}\right) \\
& =\sigma_{1} \operatorname{tr}\left(\left(I_{p}+r A\right)^{-1}\left(X_{1}^{\prime} Q_{1} X_{1}\right)^{-1}\right) \\
& =\sigma_{1} \operatorname{tr}\left(\left(I_{p}+r \Delta\right)^{-1} K_{2} K_{1}^{\prime} \Sigma^{-1} K_{1} K_{2}^{\prime}\right) \\
& =\sigma_{1} \operatorname{tr}\left(\left(I_{p}+r \Delta\right)^{-1} K^{\prime} \Sigma^{-1} K\right),
\end{aligned}
$$

where $K=K_{1} K_{2}^{\prime}$ is an orthogonal matrix. Thus we have

$$
\begin{aligned}
e_{3}\left(\beta^{*}(r) \mid \hat{\beta}\right) & =\frac{\operatorname{tr}\left(\operatorname{Cov}\left(\beta^{*}(r)\right)\right)}{\operatorname{tr}(\operatorname{Cov}(\hat{\beta}))} \\
& =\frac{\operatorname{tr}\left(\left(I_{p}+r \Delta\right)^{-1} K^{\prime} \Sigma^{-1} K\right)}{\sum_{i=1}^{p} \theta_{i}^{-1}} .
\end{aligned}
$$


Using Lemma 3.2, we have

$$
\begin{aligned}
\sum_{i=1}^{p} \theta_{p+1-i}^{-1}\left(1+r \zeta_{i}\right)^{-1} & \leq \operatorname{tr}\left(\left(I_{p}+r \Delta\right)^{-1} K^{\prime} \Sigma^{-1} K\right) \\
& \leq \sum_{i=1}^{p} \theta_{p+1-i}^{-1}\left(1+r \zeta_{p+1-i}\right)^{-1}
\end{aligned}
$$

Thus

$$
\frac{\sum_{i=1}^{p} \theta_{p+1-i}^{-1}\left(1+r \zeta_{i}\right)^{-1}}{\sum_{i=1}^{p} \theta_{i}^{-1}} \leq e_{3}\left(\beta^{*}(r) \mid \hat{\beta}\right) \leq \frac{\sum_{i=1}^{p} \theta_{p+1-i}^{-1}\left(1+r \zeta_{p+1-i}\right)^{-1}}{\sum_{i=1}^{p} \theta_{i}^{-1}}
$$

Corollary 4.1 Let $\beta^{*}(r)$ and $\hat{\beta}$ be given in Equations (7) and (3), let $e_{3}\left(\beta^{*}(r) \mid \hat{\beta}\right)$ be defined in Equation (16), $X_{1}^{\prime} Q_{1} X_{1}$ and $X_{2}^{\prime} Q_{2} X_{2}$ communicate, then we have

$$
\frac{\theta_{p}}{\theta_{1}+r \eta_{1}} \leq e_{3}\left(\beta^{*}(r) \mid \hat{\beta}\right) \leq \frac{\theta_{1}}{\theta_{p}+r \eta_{p}}
$$

where $\theta_{1} \geq \cdots \geq \theta_{p}$ is the ordered eigenvalues of $X_{1}^{\prime} Q_{1} X_{1}, \eta_{1} \geq \cdots \geq \eta_{p}$ is the ordered eigenvalues of $X_{2}^{\prime} Q_{2} X_{2}$.

Proof Since $X_{1}^{\prime} Q_{1} X_{1}$ and $X_{2}^{\prime} Q_{2} X_{2}$ communicate, there exists an orthogonal matrix $G$ such that

$$
\begin{aligned}
& G^{\prime} X_{1}^{\prime} Q_{1} X_{1} G=\operatorname{diag}\left(\theta_{1}, \ldots, \theta_{p}\right)=\Sigma, \\
& G^{\prime} X_{2}^{\prime} Q_{2} X_{2} G=\operatorname{diag}\left(\eta_{1}, \ldots, \eta_{p}\right)=\Omega,
\end{aligned}
$$

where $\theta_{1} \geq \cdots \geq \theta_{p}$ is the ordered eigenvalues of $X_{1}^{\prime} Q_{1} X_{1}, \eta_{1} \geq \cdots \geq \eta_{p}$ is the ordered eigenvalues of $X_{2}^{\prime} Q_{2} X_{2}$.

By the definition of $e_{3}\left(\beta^{*}(r) \mid \hat{\beta}\right)$, we have

$$
\begin{aligned}
e_{3}\left(\beta^{*}(r) \mid \hat{\beta}\right) & =\frac{\operatorname{tr}\left(\operatorname{Cov}\left(\beta^{*}(r)\right)\right)}{\operatorname{tr}(\operatorname{Cov}(\hat{\beta}))} \\
& =\frac{\sum_{i=1}^{p}\left(\theta_{i}+r \eta_{i}\right)^{-1}}{\sum_{i=1}^{p} \theta_{i}^{-1}} .
\end{aligned}
$$

Thus we have

$$
\frac{\theta_{p}}{\theta_{1}+r \eta_{1}} \leq e_{3}\left(\beta^{*}(r) \mid \hat{\beta}\right) \leq \frac{\theta_{1}}{\theta_{p}+r \eta_{p}} .
$$

Then we can give the lower and upper bounds of $e_{4}\left(\beta^{*}(r) \mid \tilde{\beta}\right)$.

Theorem 4.2 Let $\beta^{*}(r)$ and $\tilde{\beta}$ be given in Equations (7) and (5), let $e_{4}\left(\beta^{*}(r) \mid \tilde{\beta}\right)$ be defined in Equation (17), then we have

$$
\frac{\sum_{i=1}^{p} \eta_{p+1-i}^{-1}\left(r+\iota_{i}\right)^{-1}}{\sum_{i=1}^{p} \eta_{i}^{-1}} \leq e_{4}\left(\beta^{*}(r) \mid \tilde{\beta}\right) \leq \frac{\sum_{i=1}^{p} \eta_{p+1-i}^{-1}\left(r+\iota_{p+1-i}\right)^{-1}}{\sum_{i=1}^{p} \eta_{i}^{-1}},
$$


where $\eta_{1} \geq \cdots \geq \eta_{p}$ is the ordered eigenvalues of $X_{2}^{\prime} Q_{2} X_{2}, \iota_{1} \geq \cdots \geq \iota_{p}$ is the ordered eigenvalues of $\left(X_{2}^{\prime} Q_{2} X_{2}\right)^{-1 / 2}\left(X_{1}^{\prime} Q_{1} X_{1}\right)\left(X_{2}^{\prime} Q_{2} X_{2}\right)^{-1 / 2}$.

Corollary 4.2 Let $\beta^{*}(r)$ and $\tilde{\beta}$ be given in Equations (7) and (5), let $e_{4}\left(\beta^{*}(r) \mid \tilde{\beta}\right)$ be defined in Equation (17), $X_{1}^{\prime} Q_{1} X_{1}$ and $X_{2}^{\prime} Q_{2} X_{2}$ communicate, then we have

$$
\frac{\eta_{p}}{\theta_{1}+r \eta_{1}} \leq e_{4}\left(\beta^{*}(r) \mid \tilde{\beta}\right) \leq \frac{\eta_{1}}{\theta_{p}+r \eta_{p}},
$$

where $\theta_{1} \geq \cdots \geq \theta_{p}$ is the ordered eigenvalues of $X_{1}^{\prime} Q_{1} X_{1}, \eta_{1} \geq \cdots \geq \eta_{p}$ is the ordered eigenvalues of $X_{2}^{\prime} Q_{2} X_{2}$.

\section{Concluding remarks}

In this article, we have introduced four relative efficiencies in two linear regression equations system with identical parameter vectors, and we have also given the lower and upper bounds for the four relative efficiencies.

\section{Competing interests}

The author declares that they have no competing interests.

\section{Acknowledgements}

This work was supported by the Scientific Research Foundation of Chongqing University of Arts and Sciences (Grant No. R2013SC12), Program for Innovation Team Building at Institutions of Higher Education in Chongqing (Grant No. KJTD201321), and the National Natural Science Foundation of China (Grant Nos. 71271227, 11201505).

Received: 23 March 2014 Accepted: 3 July 2014 Published: 15 August 2014

\section{References}

1. Liu, AY: Estimation of the parameters in two linear models with only some of the parameter vectors identical. Stat. Probab. Lett. 29, 369-375 (1996)

2. Ma, TF, Wang, SG: Estimation of the parameters in a two linear regression equations system with identical parameter vectors. Stat. Probab. Lett. 79, 1135-1140 (2009)

3. Yang, $\mathrm{H}$ : Extensions of the Kantorovich inequality and the error ratio efficiency of the mean square. Math. Appl. 4, 85-90 (1988)

4. Wang, SG, Ip, WC: A matrix version of the Wielandt inequality and its applications to statistics. Linear Algebra Appl. 296, 171-181 (1999)

5. Liu, SZ: Efficiency comparisons between the OLSE and the BLUE in a singular linear model. J. Stat. Plan. Inference 84 $191-200(2000)$

6. Liu, SZ, Lu, CY, Puntanen, S: Matrix trace Wielandt inequalities with statistical applications. J. Stat. Plan. Inference 139 2254-2260 (2009)

7. Yang, H, Wang, LT: An alternative form of the Watson efficiency. J. Stat. Plan. Inference 139, 2767-2774 (2009)

8. Wang, LT, Yang, H: Several matrix Euclidean norm inequalities involving Kantorovich inequality. J. Inequal. Appl. 2009, Article ID 291984 (2009)

9. Wang, LT, Yang, H: Matrix Euclidean norm Wielandt inequalities and their applications to statistics. Stat. Pap. 53, 521-530 (2012)

10. Yang, H, Wu, JB: Some matrix norm Kantorovich inequalities and their applications. Commun. Stat., Theory Methods 22, 4078-4085 (2011)

11. Wang, SG, Jia, ZZ: Matrix Inequality. Anhui Education Press, Hefei (1994)

12. Yuan, JC: Relative efficiencies of mixed estimator with respect to LS estimator in linear regression model. J. China Univ. Sci. Technol. 30, 285-291 (2000) 